\title{
Shape of plasmon bands at the Brillouin-zone boundary
}

\author{
K. Sturm \\ Institut für Festkörperforschung, Forschungszentrum Jülich G.m.b.H., D-5170 Jülich, Federal Republic of Germany \\ W. Schülke \\ Institut für Physik, Universität Dortmund, D-4600 Dortmund 50, Federal Republic of Germany
}

(Received 7 April 1992)

\begin{abstract}
Recently an off-diagonal element of the density response function was measured by inelastic scattering of $\mathrm{x}$ rays from standing wave fields (coherent inelastic $\mathrm{x}$-ray scattering). The observed peak-valley structure demonstrated the existence of plasmon bands separated by a plasmon band gap for scattering vectors $\mathbf{k}$ close to the $L$ point of the Brillouin zone in $\mathrm{Si}$. In standard inelastic x-ray scattering spectroscopy which yields the diagonal density response the plasmon band gap is obscured by strong line broadening of the two plasmons resulting in a single broad peak. Here we explain that by measuring both the diagonal and the off-diagonal response for $\mathbf{k}=\mathbf{G}_{111} / 2$ the line shapes of the two plasmons at the Brillouinzone boundary can be reconstructed. Theoretical results obtained from a $2 \times 2$ effective dielectric matrix evaluated to second order in the local pseudopotential are in very good qualitative agreement with experiment.
\end{abstract}

Some time ago the existence of plasmon bands and, in particular, of sizable plasmon band gaps at the Brillouinzone (BZ) boundary, evaluated to first order in the relevant pseudopotential coefficient, were predicted in semiconductors. ${ }^{1}$ (In most simple metals the plasmon cutoff wave number $k_{c}$ is inside the BZ.) However, neither in electron-energy-loss spectroscopy (EELS) nor in standard inelastic $x$-ray scattering spectroscopy were any signs of a double peak structure and hence any plasmon band gap detected. Later this failure was explained theoretically ${ }^{2}$ by strong line broadening due to interband transitions and local field effects. In addition a considerable reduction of the first-order plasmon band gap in second-order perturbation theory was predicted. It had been demonstrated before that second-order perturbation theory yielded a description of the plasmon line shape in the long-wavelength limit in very good agreement with experiments. $^{3}$

Very recently ${ }^{4}$ it was shown that by using the superposition of an incident and a Bragg-reflected wave within the crystal to act as initial photon state of an inelastic $x$ ray scattering experiment [coherent inelastic $x$-ray scattering ${ }^{5}$ (CIXS)] the double differential scattering cross section is basically composed of two terms. One is directly connected with the diagonal element of the response function, the other one, an interference term, is mainly determined by an off-diagonal element, where the $\mathbf{G}$ vector involved corresponds to the Bragg reflection which defines the initial photon state. The weight and sign of this interference term can be tuned by the angular deviation from the exact Bragg position in such a way that its contribution could be separated experimentally. It was explained that the peak-valley structure of the offdiagonal element observed in this way is indicative of two plasmons at the BZ boundary.

Here we wish to point out that in addition for $\mathbf{k}=\mathbf{G}_{111} / 2$ the shape of the two plasmons can be easily reconstructed from the measured diagonal and off- diagonal response functions.

Bragg reflection of plasmons in a crystal leading to plasmon band splitting at the BZ boundary in a way similar to that in ordinary band-structure theory is approximately accounted for in a so-called two-plasmon-band model $^{2}$ which is based on an effective $2 \times 2$ dielectric matrix whose elements are evaluated to second order in the (local) pseudopotential. Within this model the offdiagonal element of the response function that is obtained by CIXS (Ref. 4) is given by

$$
\begin{aligned}
\operatorname{Im}[ & \left.-\widetilde{\epsilon}^{-1}(\mathbf{k}, \omega)\right]_{0 G} \\
& =\operatorname{Im}\left(\frac{\widetilde{\epsilon}_{0 \mathbf{G}}(\mathbf{k}, \omega)}{\tilde{\boldsymbol{\epsilon}}_{00}(\mathbf{k}, \omega) \widetilde{\boldsymbol{\epsilon}}_{\mathbf{G G}}(\mathbf{k}, \omega)-\boldsymbol{\epsilon}_{0 \mathbf{G}}(\mathbf{k}, \omega) \boldsymbol{\epsilon}_{\mathbf{G O}}(\mathbf{k}, \omega)}\right)
\end{aligned}
$$

and the diagonal density response function by

$$
\begin{aligned}
& \operatorname{Im}\left[-\widetilde{\boldsymbol{\epsilon}}^{-1}(\mathbf{k}, \omega)\right]_{00} \\
& \quad=\operatorname{Im}\left(\frac{-\widetilde{\boldsymbol{\epsilon}}_{\mathbf{G G}}(\mathbf{k}, \omega)}{\widetilde{\boldsymbol{\epsilon}}_{\mathbf{0 0}}(\mathbf{k}, \omega) \widetilde{\boldsymbol{\epsilon}}_{\mathbf{G G}}(\mathbf{k}, \omega)-\widetilde{\boldsymbol{\epsilon}}_{\mathbf{O G}}(\mathbf{k}, \omega) \widetilde{\boldsymbol{\epsilon}}_{\mathbf{G 0}}(\mathbf{k}, \omega)}\right) .
\end{aligned}
$$

Here $\left[-\widetilde{\boldsymbol{\epsilon}}^{-1}(\mathbf{k}, \omega)\right]_{\mathbf{G G}^{\prime}}$ is the $\mathbf{G G}^{\prime}$ element of inverse of the effective dielectric matrix defined by

$\widetilde{\epsilon}_{\mathrm{GG}^{\prime}}(\mathbf{k}, \omega)=\epsilon_{\mathrm{GG}^{\prime}}(\mathbf{k}, \omega)-\sum_{\mathbf{G}^{\prime \prime}} \frac{\epsilon_{\mathrm{GG}^{\prime \prime}}(\mathbf{k}, \omega) \epsilon_{\mathrm{G}^{\prime \prime} \mathrm{G}^{\prime}}(\mathbf{k}, \omega)}{\epsilon_{L}\left(\left|\mathbf{k}+\mathbf{G}^{\prime \prime}\right|, \omega\right)}$,

where the reciprocal lattice vectors $\mathbf{G}$ and $\mathbf{G}^{\prime}$ can be either 0 or $-\mathbf{G}_{111}$ and $\Sigma^{\prime \prime}$ implies that the sum extends over all reciprocal lattice vectors $G^{\prime \prime}$ except $G^{\prime \prime}=0$ and $\mathbf{G}^{\prime \prime}=-\mathbf{G}_{111}$. The elements of the dielectric matrix ${ }^{6}$ entering (3) are defined by

$$
\epsilon_{\mathbf{G G}^{\prime}}(\mathbf{k}, \omega)=\delta_{\mathbf{G G}^{\prime}}-v_{\mathbf{k}+\mathbf{G}} \chi^{0}\left(\mathbf{k}+\mathbf{G}, \mathbf{k}+\mathbf{G}^{\prime} ; \omega\right) .
$$

$v_{\mathbf{k}+\mathbf{G}}=4 \pi e^{2} /|\mathbf{k}+\mathbf{G}|^{2}, \quad \chi^{0}\left(\mathbf{k}+\mathbf{G}, \mathbf{k}+\mathbf{G}^{\prime} ; \omega\right)$ is the independent particle density-density response function, and 


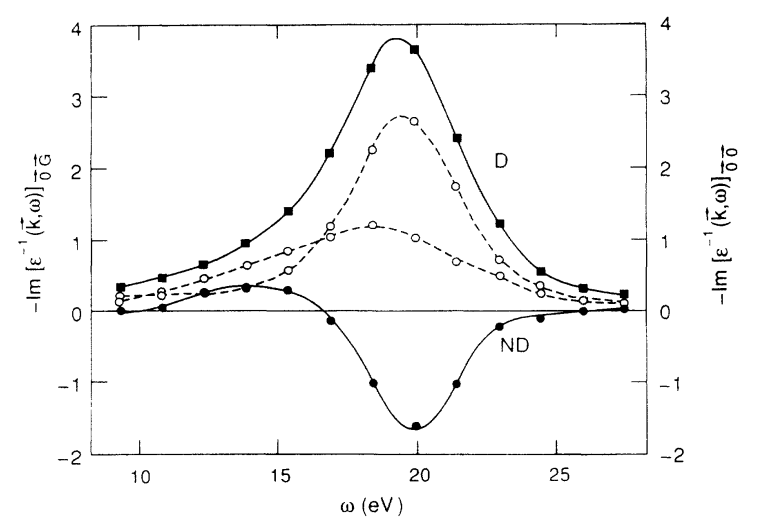

FIG. 1. Experiment: Construction of the zone boundary plasmons from the measured diagonal (D) and nondiagonal (ND) response functions of Ref. 4 according to Eqs. (8) and (9), respectively.

$\epsilon_{L}(|\mathbf{k}+\mathbf{G}|, \omega)$ is the well-known Lindhard dielectric function of the homogeneous electron gas.

For $\mathbf{k}$ at the [111] $\mathrm{BZ}$ face, more precisely for $\mathbf{k}=\mathbf{G}_{111} / 2$, the shape of the two plasmons can be reconstructed from the diagonal and off-diagonal response functions. Because of inversion symmetry (applicable to Si) we have (with $\mathbf{G} \equiv-\mathbf{G}_{111}$ )

$$
\boldsymbol{\epsilon}_{\mathrm{GG}}\left(\mathbf{G}_{111} / 2, \omega\right)=\boldsymbol{\epsilon}_{00}\left(\mathbf{G}_{111} / 2, \omega\right)
$$

and

$$
\widetilde{\epsilon}_{0 G}\left(\mathbf{G}_{111} / 2, \omega\right)=\widetilde{\epsilon}_{\mathrm{G} 0}\left(\mathbf{G}_{111} / 2, \omega\right) .
$$

Then, suppressing the arguments $\mathbf{k}=\mathbf{G}_{111} / 2$ and $\omega$, Eqs. (1) and (2) can be written in the form

$$
\operatorname{Im}\left[-\widetilde{\epsilon}^{-1}\right]_{O G}=\frac{1}{2}\left[\operatorname{Im}\left(\frac{-1}{\tilde{\epsilon}_{00}+\widetilde{\epsilon}_{0 G}}\right)-\operatorname{Im}\left(\frac{-1}{\boldsymbol{\epsilon}_{00}-\widetilde{\epsilon}_{0 G}}\right)\right]
$$

and

$$
\operatorname{Im}\left[-\widetilde{\epsilon}^{-1}\right]_{00}=\frac{1}{2}\left[\operatorname{Im}\left(\frac{-1}{\widetilde{\epsilon}_{00}+\widetilde{\epsilon}_{0 G}}\right)+\operatorname{Im}\left(\frac{-1}{\boldsymbol{\epsilon}_{00}-\boldsymbol{\epsilon}_{0 G}}\right)\right] .
$$

The main result is that while the diagonal response function is made up by the sum of the two plasmon lines the off-diagonal response function is determined by the difference of the two plasmon lines leading to a peakvalley structure. Obviously Eqs. (6) and (7) allow a simple reconstruction of the two zone boundary plasmons from the measured diagonal and off-diagonal response functions, i.e.,

$$
\begin{aligned}
& \frac{1}{2} \operatorname{Im}\left[\frac{-1}{\widetilde{\epsilon}_{00}+\widetilde{\epsilon}_{0 G}}\right]=\frac{1}{2}\left\{\operatorname{Im}\left[-\widetilde{\epsilon}^{-1}\right]_{00}+\operatorname{Im}\left[-\widetilde{\epsilon}^{-1}\right]_{O G}\right\}, \\
& \frac{1}{2} \operatorname{Im}\left[\frac{-1}{\widetilde{\epsilon}_{00}-\widetilde{\epsilon}_{0 G}}\right]=\frac{1}{2}\left\{\operatorname{Im}\left[-\tau^{-1}\right]_{00}-\operatorname{Im}\left[-\boldsymbol{\epsilon}^{-1}\right]_{O G}\right\} .
\end{aligned}
$$

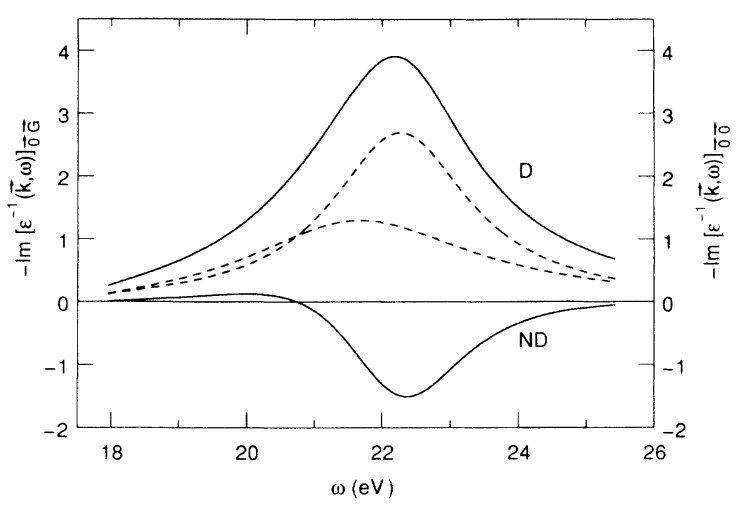

FIG. 2. Theory: D: Diagonal response function (solid line) made up by the superposition of the two zone boundary plasmons (dashed lines) according to Eq. (7) at $\mathbf{k}=\mathbf{G}_{111} / 2$. ND: Nondiagonal response function resulting from the difference of the zone boundary plasmons according to Eq. (6).

In Fig. 1 the two plasmon lines close to the $L$ point (open circles) are constructed according to Eqs. (8) and (9) from the measured diagonal and off-diagonal response functions (full squares and full circles, respectively). ${ }^{7}$ The lines are drawn to bring out the shape of the curves more clearly.

Within the nearly free-electron approximation $\epsilon_{00}$ and $\epsilon_{O G}$ can be calculated analytically to second order in the pseudopotential (for details, see Ref. 2). Using for simplicity a local pseudopotential ${ }^{8}$ the theory is applied to $\mathrm{Si}$ and the diagonal and off-diagonal response functions are determined as depicted in Fig. 2. The superposition of the two plasmons (dashed curves) results in a single peak (solid line), while the difference exhibits the peak-valley structure which has been observed by CIXS. There is very good qualitative agreement between theory and experiment. Especially, the much larger width of the firstband plasmon compared to the second-band plasmon, as has already been predicted by Daling, van Haeringen, and Farid, ${ }^{9}$ is evident in theory and experiment. Theoretically the curves are shifted to higher frequencies, a wellknown deficiency of the random-phase approximation used here. Inclusion of exchange-correlation in terms of the local field factor proposed by Utsumi and Ichimaru ${ }^{10}$ which satisfies various requirements in the small and large $q$ limits yields a minor improvement only. The larger width of the experimental peaks compared to the calculated ones is mainly due to the limited energy resolution, which has been adjusted to $2.5 \mathrm{eV}$ (see Ref. 4).

In summary we have shown that on the basis of the dielectric response formalism a detailed understanding of the plasmon bands at the $\mathrm{BZ}$ boundary can be obtained from diagonal and off-diagonal response functions, which can be measured in coherent inelastic $x$-ray scattering.

This work has been partly funded by the German Federal Ministry of Research and Technology (BMFT) under Contract No. 05434 AXB. 
${ }^{1}$ R. Girlanda, M. Parinello, and E. Tosatti, Phys. Rev. Lett. 36, 1386 (1976).

${ }^{2}$ L. E. Oliveira and K. Sturm, Phys. Rev. B 22, 6283 (1980). Note that in the evaluation of the second-order contribution to the imaginary part of $\epsilon_{0 G}$ a sign error occurred that is responsible for the rather similar heights of the plasmons at the $L$ point as shown in Fig. 4 . When corrected it leads to the results shown in Fig. 2 of the present paper.

${ }^{3}$ K. Sturm, Phys. Rev. Lett. 40, 1599 (1978).

${ }^{4}$ W. Schülke and A. Kaprolat, Phys. Rev. Lett. 67, 879 (1991).
${ }^{5}$ W. Schülke, Solid State Commun. 43, 863 (1982); 44, 1130 (1982).

${ }^{6}$ N. Wiser, Phys. Rev. 129, 62 (1963).

${ }^{7}$ Note that the energy scale of Fig. 2 in Ref. 4 is too large by $9 \%$ due to a scaling error.

8J. R. Chelikowsky and M. L. Cohen, Phys. Rev. B 10, 5095 (1974).

${ }^{9}$ R. Daling, W. van Haeringen, and B. Farid, Phys. Rev. B 44, 2952 (1991).

${ }^{10}$ K. Utsumi and S. Ichimaru, Phys. Rev. A 26, 603 (1982). 